CASE REPORT

\title{
Systemic absorption of FD\&C blue dye associated with patient mortality
}

\author{
S Gaur, T Sorg, V Shukla
}

Postgrad Med J 2003;79:602-603

The addition of FD\&C blue dye to enteral feeds is a common practice in hospitals to detect aspiration. However, the degree of systemic absorption and safety of this dye in critically ill patients has not been studied. A patient with sepsis who died after systemic absorption of FD\&C blue dye Nol is described.

$\mathrm{F}$ D\&C blue dye No 1 is a triphenylmethane dye approved by the Food and Drug Administration for use in food after initial experiments in animals showed it to be relatively non-toxic. ${ }^{1}$ In normal subjects less than $5 \%$ of the dye is absorbed systemically, the majority being excreted in the stools. However the predominant use of this dye in hospitals is in identifying aspiration in critically ill patients. Such patients have increased gastrointestinal permeability secondary to enterocyte death and stress induced release of neuroendocrine factors in the intestinal epithelium. ${ }^{2}$ There are no data about the extent of systemic absorption and safety of this dye under such conditions.

\section{CASE REPORT}

A 56 year old African-American woman was transferred to our hospital from a nursing home after she became increasingly lethargic. Three months previously she had been diagnosed with a neuromuscular disorder impairing her ability to swallow. She had a gastrostomy tube placed and was receiving enteral nutrition. She had recently completed a course of antibiotics for pneumonia. Four days before admission she had started having multiple episodes of loose stools with abdominal cramps. At the time of initial evaluation she was found to have a blood pressure of 70 / $40 \mathrm{~mm} \mathrm{Hg}$, temperature of $39^{\circ} \mathrm{C}$, and a pulse rate of 118 beats/min. She was lethargic and responded only to painful stimuli. Her Glasgow coma scale score was 8. A Foleys catheter was in place draining turbid urine; her lungs were clear to auscultation. There was no erythema or tenderness at the gastrostomy site. The rest of the physical examination was unremarkable.

Laboratory evaluation disclosed the following: bicarbonate $27 \mathrm{mmol} / \mathrm{l}(22-26)$, potassium $3.1 \mathrm{mmol} / \mathrm{l}(3.5-5)$, sodium $146 \mathrm{mmol} / \mathrm{l}$ (138-144), chloride $93 \mathrm{mmol} / \mathrm{l}$ (98-107), blood urea nitrogen $13.5 \mathrm{mmol} / \mathrm{l}(2.9-7.1)$, and serum creatinine $62.1 \mu \mathrm{mol} / \mathrm{l}(61.9-115)$. Haematological analysis revealed a white cell count of $16.8 \times 10^{9} / 1(4-11)$ with $92 \%$ neutrophils, a platelet count of $440 \times 10^{9} / 1(150-450)$, and a haemoglobin of $101 \mathrm{~g} / \mathrm{l}(120-160)$. A plain film of her abdomen showed a non-specific bowel pattern, and chest radiography was negative for infiltrates. She was treated with intravenous fluids, broad spectrum antibiotics, and required vasopressor support. Cultures drawn at admission grew Klebsiella pneumoniae and Candida albicans in her urine and enterococi in her blood stream. Stool specimens were positive for Clostridium difficile toxin. Based on these, antibiotics were changed to intravenous ampicillin, fluconazole, metronidazole, and ciprofloxacin. Enteral nutrition was continued. With antibiotics and fluids the patient improved and became more alert.

On the second day of admission vasopressors were discontinued and she remained haemodynamically stable. Fever, however, persisted and repeat radiography of her chest on the fourth day of hospitalisation showed a new infiltrate in the right middle lobe with air bronchograms. Ampicillin and ciproflaxcin were discontinued and she was started on intravenous vancomycin and cefepime. Metronidazole and fluconazole were continued. Supplemental oxygen was given at a rate of $3 \mathrm{l} / \mathrm{min}$.

As there was a concern that she might be aspirating, FD\&C blue dye No 1 was added to her enteral feeds. The next day her skin, mucus membranes, and urine turned greenish blue. Her vital signs showed a blood pressure of $140 / 92 \mathrm{~mm} \mathrm{Hg}$, respiratory rate of 22 breaths/min, and temperature of $37^{\circ} \mathrm{C}$. Multiple blisters were noted on her abdomen and lower limbs. Fluid within the blisters was also green.

Arterial blood gas analysis on three litres of oxygen showed a pH of 7.31, carbon dioxide pressure of $4 \mathrm{kPa}$, and oxygen pressure of $12.8 \mathrm{kPa}$. Biochemistry was as follows: serum bicarbonate $15 \mathrm{mmol} / \mathrm{l}(22-26)$, serum potassium $5 \mathrm{mmol} / \mathrm{l}$ (3.5-5), serum chloride $100 \mathrm{mmol} / \mathrm{l}$ (98-107), serum sodium $138 \mathrm{mmol} / \mathrm{l}$ (138-144), and serum lactate level $5.2 \mathrm{mmol} / \mathrm{l} \quad(0.5-2.2)$. She became progressively more lethargic, acidotic, and died six days later. At necropsy there was greenish blue discoloration of all her internal organs.

\section{DISCUSSION}

Maloney et al have previously reported two deaths in association with systemic absorption of blue dye No $1 .^{3}$ The first patient was a 54 year old woman with chronic renal and congestive heart failure who developed staphylococcal pneumonia with sepsis. Two days after adding FD\&C blue dye No 1 to her enteral feeds, her skin and serum turned green, and she died of refractory hypotension and acidosis. The second patient was a 12 month old boy with trisomy 21 who underwent a tracheostomy for obstructive apnoea. He developed pseudomonas pneumonia and blue dye No 1 was added to enteral feeds to detect aspiration. The next day his skin, serum, and urine turned blue and he too died of refractory hypotension and acidosis. Our case report provides additional evidence associating systemic absorption of this dye with mortality in critically ill patients. All three were

Abbreviations: ADP, adenosine diphosphate; ATP, adenosine triphosphate 
haemodynamically stable until systemic absorption of the dye occurred heralded by discoloration of the skin and serum.

The dye has been shown to interfere with the action of the adenosine triphosphate (ATP)/adenosine diphosphate (ADP) translocator situated in the inner mitochondrial membrane. ${ }^{45}$ This translocator is responsible for transporting ADP into the mitochondria where it is converted to ATP by the enzyme ATP synthase driven by the electron transport chain. Newly synthesised ATP is then transported to the cytoplasm for utilisation. The inhibitory effect of the dye on this translocator may be responsible for the refractory acidosis noted in all three patients. While it is difficult to ascribe the mortality solely to the dye in these critically ill patients, it is conceivable that the systemic absorption of the dye played a part in their clinical deterioration. We urge physicians to be cognisant of these facts and to use alternative methods to detect aspiration. The detection of glucose in tracheobronchial secretions using glucose oxidase reagent strips is a more sensitive and a safer alternative. $^{6}$
Authors' affiliations

S Gaur, T Sorg, V Shukla, Department of Internal Medicine, Wright State University, School Of Medicine, 3640 Colonel Glenn Highway, Dayton, Ohio, USA

Correspondence to: Dr Gaur; sumitgaurmd@hotmail.com

Submitted 28 December 2002

Accepted 11 February 2003

\section{REFERENCES}

1 Food and Drug Administration. FD\&C blue no 1. Federal Register 1982;47:42563-6.

2 Soderholm JD, Perdue MH. Stress and the gastrointestinal tract: II. Stress and intestinal barrier function. Am J Physiol 2001 ;280:7G-13.

3 Maloney JP, Halbower AC, Fagan KA, et al. Systemic absorption of food dye in patients with sepsis. N Engl J Med 2000;343:1047-8.

4 Reyes FG, Valim MF, Vercesi AE. Effect of organic synthetic food colors on mitochondrial respiration. Food Additives and Contaminants 1996;13:5-11.

5 Noda K, Yoshimoto M, Hatano S, et al. Effect of coal tar dyes on oxygen uptake in mitochondria isolated from rat liver. Journal of the Food Hygienic Society of Japan 1985;26:203-7.

6 Metheny NA, Clouse RE. Bedside methods for detecting aspiration in tube-fed patients. Chest 1997;111:724-31.

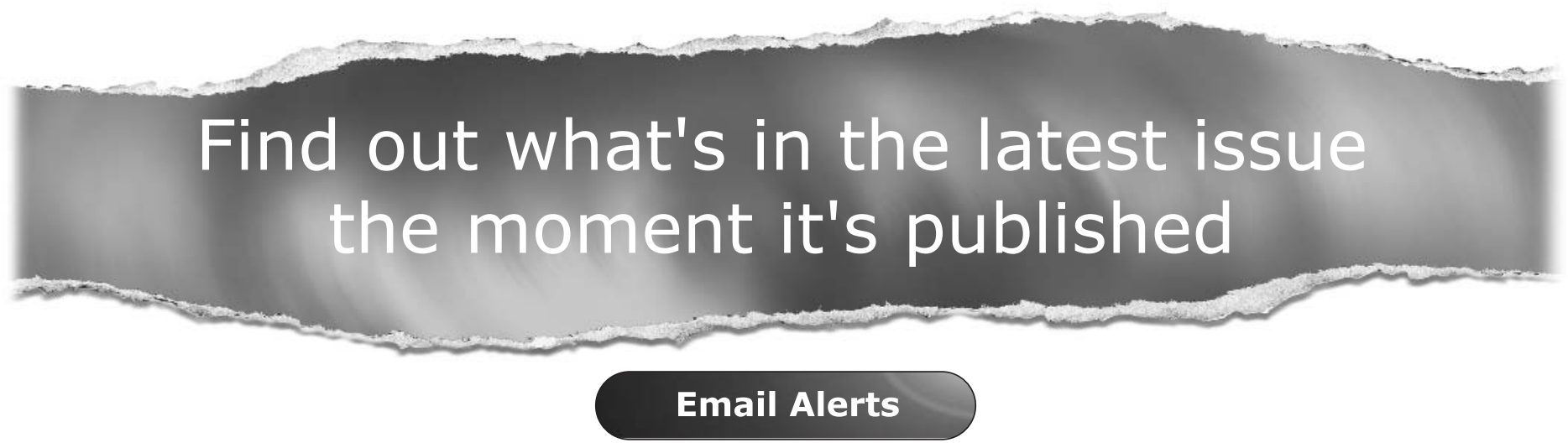

Sign up to receive the table of contents by email every month. You can select from three alerts: Table of Contents (full), TOC Awareness (notice only); Postgraduate Medical Journal related announcements.

www.postgradmedj.com 